\section{Comment "On the Statistics of the Product of a Gaussian Process and a Pseudo Random Binary Code"}

In a recent correspondence, Painter ${ }^{1}$ shows that the first-order statistics of the product of a Gaussian noise process and a binary process taking on only the values \pm 1 is Gaussian. The purpose of the present letter is to point out that the product process, although having Gaussian first-order statistics, is not a Gaussian process; i.e., the higher-order statistics are not in general multivariate Gaussian.

To show this, consider $Z_{1}=C_{1} X_{1}$ and $Z_{2}=C_{2} X_{2}$ where $X_{1}, X_{2}$ are bivariate Gaussian random variables, and $C_{1}, C_{2}$ take on the values \pm 1 (independent of $\left.X_{1}, X_{2}\right)$ with joint probabilities $\alpha(1,1), \alpha(1,-1)$, $\alpha(-1,1), \alpha(-1,-1)$. The characteristic function of the distribution of $Z_{1}, Z_{2}$ is given by

$$
\begin{aligned}
M_{z}\left(u_{1}, u_{2}\right)= & \left(\exp \left[i\left(u_{1} Z_{1}+u_{2} Z_{2}\right)\right]\right. \\
= & \alpha(1,1) M_{x}\left(u_{1}, u_{2}\right)+\alpha(1,-1) M_{x}\left(u_{1},-u_{2}\right) \\
& +\alpha(-1,1) M_{x}\left(-u_{1}, u_{2}\right)+\alpha(-1,-1) M_{x}\left(-u_{1},-u_{2}\right)
\end{aligned}
$$

where $M_{x}\left(u_{1}, u_{2}\right)$ is the characteristic function of the distribution of $X_{1}, X_{2}$. If $X_{1}$ and $X_{2}$ are assumed to have zero mean, unit variance, and covariance $\rho$, then ${ }^{2}$

$$
M_{x}\left(u_{1}, u_{2}\right)=\exp \left[-\frac{1}{2}\left(u_{1}^{2}+2 \rho u_{1} u_{2}+u_{2}^{2}\right)\right]
$$

so that

$$
\begin{aligned}
M_{2}\left(u_{1}, u_{2}\right)= & {\left[a \exp \left(-\rho u_{1} u_{2}\right)+(1-a) \exp \left(\rho u_{1} u_{2}\right)\right] } \\
& \times\left[\exp -\frac{1}{2}\left(u_{1}^{2}+u_{2}^{2}\right)\right]
\end{aligned}
$$

where

$$
a=\alpha(1,1)+\alpha(-1,-1)=1-[\alpha(1,-1)+\alpha(-1,1)] .
$$

Equation (3) is the characteristic function of a linear combination of two bivariate Gaussian distributions with different covariances. Such a linear combination is not generally bivariate Gaussian. [A necessary condition $^{2}$ for $Z_{1}, Z_{2}$, to be bivariate Gaussian is that $M_{z}\left(u_{1}, u_{2}\right)$ be expressible as the exponential of a quadratic form in $u_{1}$ and $u_{2}$. For $\rho \neq 0,(3)$ is not expressible in the requisite form. ]

Note that for the single variable case

$$
M_{s}(u)=\alpha(1) M_{x}(u)+\alpha(-1) M_{x}(-u)=M_{x}(u)
$$

since $M_{x}(u)=M_{x}(-u)$ and $\alpha(1)+\alpha(-1)=1$. Hence the first-order statistics are indeed Gaussian, as Painter shows by a somewhat more elaborate calculation.

Thus given $Z(t)=C(t) X(t)$, although $Z\left(t_{1}\right)$ is Gaussian, $Z\left(t_{1}\right)$, $Z\left(t_{2}\right)$ are not in general jointly Gaussian. Hence $Z(t)$ is not a Gaussian process. This result could also be inferred from continuity considerations. Consider the stationary binary process

$$
C(t)=\sum_{n=-\infty}^{\infty} c_{n} u(t-n-\theta)
$$

where $u(t)$ is the unit pulse on the interval $[0,1)$, and $\theta,\left\{c_{n}\right\}$ are independent random variables, $\theta$ being uniformly distributed on $[0,1)$, and $c_{n}$ taking on the values \pm 1 with probability $\frac{1}{3}$. The covariance of this process is readily determined to be

$$
\langle C(t) C(t+\tau)\rangle=\left\{\begin{array}{cc}
1-|\tau| & \text { for }|\tau|<1 \\
0 & \text { for }|\tau|>1
\end{array}\right.
$$

which is a continuous function of $\tau$. If the Gaussian process $X(t)$ is also assumed stationary with continuous covariance and independent of $C(t)$, then the covariance of the $Z$-process is given by the product of that of the $C$ and $X$ processes, and is continuous. However, almost all sample functions $C(t)$ contain an infinite number of jump discontinuities; hence, almost all sample functions of $Z(t)$ contain jump discontinuities. However, for a stationary Gaussian process,

Manuscript received March 2, 1966.

1 J. H. Painter, Proc. IEEE (Correspondence), vol. 53, pp. 2118-2119, December 1965.

W. B. Davenport, Jr., and W. L. Root, An Introduction to the Theory of Random Signals and Noise. New York: McGraw-Hill, 1958, ch. 8. by a theorem of Belayev, the continuity of the covariance function precludes the occurrence of jump discontinuties in almost all sample functions. Consequently, $Z(t)$ (which is stationary) cannot be a Gaussian process.

The distinction between a Gaussian process and a process having only first-order Gaussian statistics is of practical as well as theoretical importance. For example, if a Gaussian process is passed through a linear filter, the output is a Gaussian process. However, if a process with first-order Gaussian statistics is passed through a linear filter, it is not generally true that the output has even first-order Gaussian statistics. This may be illustrated by the previous example. Let $Y=Z_{1}+Z_{2}$, where $Z_{1}$ and $Z_{2}$ are each Gaussian variables, but $Z_{1}, Z_{2}$ are not jointly Gaussian. If the joint distribution corresponding to the characteristic function in (3) is employed (setting $a=\frac{1}{2}$ for simplicity), we find for the probability density of $Y$

$$
\begin{aligned}
p(y)=\frac{1}{2} \frac{1}{\sqrt{4 \pi}}\left\{\frac{1}{\sqrt{1+\rho}} \exp [\right. & \left.-\frac{y^{2}}{4(1+\rho)}\right] \\
& \left.+\frac{1}{\sqrt{1-\rho}} \exp \left[-\frac{y^{2}}{4(1-\rho)}\right]\right\} .
\end{aligned}
$$

This is not Gaussian! A linear combination of Gaussian variables is itself Gaussian, only if the variables are jointly Gaussian (or, equivalently, only if all conditional probabilities are Gaussian).

I. JACOBS Bell Telephone Labs., Inc. Whippany, N. J.

\section{Author's Replyt}

Jacobs' complementary remarks are appreciated. That the statistics treated are obviously first order was not explicitly stated. Also not stated was the primary purpose of the correspondence, that of drawing professional comment on the use of Boolean algebra as a conceptual aid in the treatment of "analog" communication problems. The comment drawn is welcomed even if not in the area expected.

J. H. Painter

Motorola Inc.

Military Electronics Div. Scottsdale, Ariz.

Y. K. Belayev, "Continuity and Holder's conditions for sample functions of stationary Gaussian processes," 1961 4th Berkeley Symp. on Mathemalical Statistics and Probability, vol. II, pp. 23-33. (I am indebted to Dr. T. T. Kadota for bringing this information to my attention, and for discussions concerning the results therein.) 4 Manuscript received March 25, 1966.

\section{Exceptionally Cost-Effective Error Control for Data Communications}

\section{INTRODUCTION}

This letter describes an almost universally applicable approach to receiving coded or $M$-ary signals. For a given high level of performance, this approach requires substantially less hardware than the most famous error correction schemes.

In many applications, especially in military communications, message reliability at reasonable expense under highly adverse conditions is far more important than exceptionally high data rates. At moderate data rates, the approach described herein provides exceptionally high message reliability for given conditions and a given quantity of hardware. For example, the device required to perform the equivalent of correcting at least 32 bit errors, regardless of

Manuscript received March 19, 1966. 